\title{
The adoptions of USEPs in identifying usability requirements in SSM-based framework: a case study
}

\begin{abstract}
Nowadays, user demand for quality software product have been increased and developers have to make sure that their products are able to meet the user's need in order to compete in the market. Usability, which is a kind of software quality, became an important factor to determine the success or failure of a software product in this challenging market. Unfortunately, lack of attention given to usability requirements especially during the early stage of software development process and therefore increase the cost of fixing usability problems and causes the final software products are poor in quality. Therefore, this paper aimed to identify usability requirements at the requirement stage of software development by using the SSM-based analysis framework. We have conducted a case study by applying a usability elicitation pattern, USEPs, into the framework. From the result of our study, it has shown that this pattern is useful to assist the stakeholders in identifying usability requirements along with the functionality of a system. Besides, we have also made some enhancement and extensions to the framework based on our findings from the study.
\end{abstract}

Keyword: Soft system methodology; Usability elicitation patterns; Usability requirements 